\title{
Optimal configuration of gamma ray machine radiosurgery units: the sphere covering subproblem
}

\author{
Leo Liberti · Nelson Maculan · Yue Zhang
}

Received: ? / Accepted: ?

\begin{abstract}
We use reformulation techniques to model and solve a complex sphere covering problem occurring in the configuration of a gamma ray machine radiotherapy equipment unit.
\end{abstract}

Keywords reformulation $\cdot$ mathematical programming $\cdot$ gamma knife $\cdot$ sphere covering

\section{Introduction}

The gamma ray machine equipment used in stereotactic radiation therapy can deliver sphereshaped "shots" (also called foci) of radiation at a brain tumor (this medical technique is known as "Gamma Knife" radiosurgery). Foci can be represented as spheres of different sizes. Four kinds of interchangeable outer collimators on a helmet device with different radii in the set $R=\{2,4,7,9\} \mathrm{mm}$ are available for irradiating the tumor region. For a target volume larger than one focus, multiple foci can be used to cover the entire target. In practice, most target volumes are treated with 1 to 15 foci $[17,10]$. The target volume (the tumor) may have an irregular shape, but for the purpose of this study it is considered to be an ellipsoid $T$ of radii $r_{T}^{x}, r_{T}^{y}, r_{T}^{z}$ smaller than $3 \mathrm{~cm}$. With present configuration technology, treatment plans can be configured up to around 10 foci. Too many foci are impracticable for clinical treatment routines. For accurate location purpose, a stereotactic head frame is screwed into the patient's skull and cannot be taken off during both the planning procedure and the treatment process. The treatment planning becomes complex and time-consuming when several foci are needed for large or irregular tumors, because any manual tuning of

L. Liberti

LIX, École Polytechnique, 91128 Palaiseau, France

E-mail: liberti@lix.polytechnique.fr

N. Maculan

COPPE - Systems Engineering, Federal University of Rio de Janeiro, P.O. Box 68511, 21941-972 Rio de Janeiro, Brazil

E-mail: maculan@cos.ufrj.br

Y. Zhang

Physics Department, Wuhan University, P.R. China 430072

E-mail: xmaseve@hotmail.com 
foci will cause dosage re-computation. It is therefore necessary to find a suitable covering of the tumor by spheres to accelerate the planning procedure.

The existing literature concerning the use of optimization techniques in Gamma Knife radiosurgery has broader scope than the problem treated in this paper, and aims to provide a complete solution to the treatment planning problem, i.e. it concerns dosages as well as positions. We refer to [10] (Sect. 3.3) for a detailed summary of existing approaches. In particular, the approach taken by Ferris and co-workers $[3,5,4,17]$ formulates linear and nonlinear programming models where the decision variables are $D_{w}\left(x_{s}, y_{s}, z_{s}, i, j, k\right)$, i.e. the dose of radiation delivered to the tumor "pixel" $(i, j, k)$ by the shot of width $w$ centered at $\left(x_{s}, y_{s}, z_{s}\right)$. The output of these formulations is a distribution given by the values of $D_{w}$; the actual sphere covering is recovered by using simulation and parameter estimation techniques. In [10], the same general approach is followed, but the problem is solved by Generalized Benders' Decomposition. This "dosage distribution" approach also found its way into related problems [18].

In this paper we focus on the sphere covering problem, which we approach from a mathematical programming point of view: that is, we define models which we then solve to optimality (or near-optimality) by means of general-purpose solver codes aimed at specific problem classes: Mixed Integer Linear Programming (MILP), convex Mixed Integer Nonlinear Programming (cMINLP) and (nonconvex) Mixed Integer Nonlinear Programming (MINLP). The problem, which involves covering an ellipsoid by spheres, is naturally cast as a MINLP; however, as is well known, solving MINLPs is much more difficult than solving MILPs. This is why we exhibit a sequence of reformulations $[1,14]$ that either transform exactly or approximate the original MINLP into a cMINLP and eventually into different types of MILPs. By formulation we mean a formal mathematical description of an optimization problem consisting of parameters, decision variables, an objective function and constraints. Roughly speaking, a reformulation of a problem $P$ is a problem $Q$ such that all the local and global optima of $P$ can be computed from those of $Q$ (see [14] for precise definitions). We remark that the problem studied in this paper is that of establishing a good sphere covering independently of the dosage problem: we thus make no claim at solving the whole treatment problem (yet).

The main aim of this paper is to describe a methodology for solving complex (nonlinear) sphere covering problems by means of mixed-integer linear programming. However, we also mean our application example to be a proof of concept: it is possible to solve MINLPs with tens of thousands of variables by employing reformulation-based techniques. We also make an auxiliary contribution in establishing some results related to the density of certain threedimensional sphere arrangements in Sect. 3.1.

In Sect. 2, we give a mathematical programming formulation of the gamma ray unit configuration problem which is close to the (informal) description given in the medical environment, but for which there are no available solution methods. In Sections 3-5, we describe a sequence of reformulations (from MINLP to cMINLP to MILP) leading to practically useful formulations. Sect. 6 presents to modifications to the original model that stem from medical considerations. Computational results are discussed in Sect. 7.

\section{Modelling the problem}

In this section we propose a formal description of the considered problem in terms of a mathematical programming formulation. 
$\left(P_{1}\right)$ Given a compact set $T \subset \mathbb{R}^{3}$, a finite set $R \subset \mathbb{R}_{+}$and an integer $n>0$, find a set $X \subset \mathbb{R}^{3}$ of at most $n$ points and an assignment $w: X \rightarrow R$ such that $T \subset$

$\cup_{x \in X} B(x, w(x))$, where $B(x, \rho) \subset \mathbb{R}^{3}$ is the ball centered at $x$ with radius $\rho$.

Problem $\left(P_{1}\right)$ can be formulated with an infinite (and uncountable) set of variables and constraints. Let $N=\{1, \ldots, n\}$ be the maximum number of spheres, $U=\{1, \ldots,|R|\}$ the index set for the radii types, $M \geq \operatorname{diam}(T)$ a sufficiently large constant and $\varepsilon>0$ a small constant; consider the following parameters:

(a) $p$ is a point in the compact set $T \subseteq \mathbb{R}^{n}$

(b) $r: U \rightarrow R$ is the sphere's radius

and decision variables:

(a) $x: N \rightarrow \mathbb{R}^{3}$ is the center of the given sphere

(b) $y: N \rightarrow\{0,1\}$ is set to 1 if the given sphere is used, 0 otherwise

(c) $u: N \times T \rightarrow\{0,1\}$ is set to 1 if the given sphere covers the specified point, 0 otherwise

(d) $w: N \times U \rightarrow\{0,1\}$ is set to 1 if the given sphere has the specified radius, 0 otherwise.

The following set of constraints correctly describes $\left(P_{1}\right)$.

$$
\begin{aligned}
\forall i \in N, p \in T \quad\left\|x_{i}-p\right\|^{2} \leq u_{i}(p) \sum_{j \in U} w_{i j} r_{j}^{2}+\left(1-u_{i}(p)\right) M^{2} \\
\forall i \in N \quad \sum_{j \in U} w_{i j}=1 \\
\forall p \in T \quad \sum_{i \in N} u_{i}(p) \geq 1 \\
\forall i \in N \quad \int_{p \in T} u_{i}(p) d p \geq \varepsilon y_{i} \\
\forall i \in N \quad \int_{p \in T} u_{i}(p) d p \leq \operatorname{Vol}(T) y_{i},
\end{aligned}
$$

where $R=\left\{r_{j}|1 \leq j \leq| U \mid\right\}$ and $\operatorname{Vol}(T)$ denotes the Lebesgue measure of $T$ in $\mathbb{R}^{3}$. Constraints (1) say that if sphere $i$ covers $p$, then the Euclidean distance between $p$ and the sphere center must be at most the sphere radius. Constraints (2) ensure that exactly one radius value is chosen for each sphere. Constraints (3) make sure that each point of $T$ is covered by at least one sphere. Constraints (4) make sure that any selected sphere covers a nonzero volume of $T$, and (5) force $u_{i}$ to be the zero function if the $i$-th sphere is not selected. This is a nonlinear, nonconvex mixed-integer infinite programming problem, for which there is no known standard solution algorithm.

\section{Approximation by discretization}

In this section we propose an approximating reformulation: it consists in replacing $T$ by a finite set of points in $T$. Consider a fixed cubic lattice $P$ of $m^{3}$ points in $\mathbb{R}^{3}$ such that conv $(P)$ is a cube of side $(m-1) \Delta$, where $\Delta>0$ is the side length of each cubic element in the lattice, $T \subseteq \operatorname{conv}(P)$, and for all $P^{\prime} \subset P$ we have $T \not \subset \operatorname{conv}\left(P^{\prime}\right)$. Essentially, $P$ is the smallest threedimensional cubic lattice with $m$ points per side that determines a cubic region containing $T$. Now consider an index set $V \subseteq\left\{1, \ldots, m^{3}\right\}$ such that $P \cap T=\left\{p_{v} \mid v \in V\right\}$ as being part of the problem input (i.e. $p_{v}$ are problem parameters). We reformulate the definition of some decision variables of $\left(P_{1}\right)$ : 
(c') $u: N \times V \rightarrow\{0,1\}$ is set to 1 if the given sphere covers the specified point.

We define $\left(P_{1}^{\prime}\right)$ to be defined by the following set of constraints:

$$
\begin{aligned}
\forall i \in N, v \in V \quad\left\|x_{i}-p_{v}\right\|^{2} \leq u_{i v} \sum_{j \in U} w_{i j} r_{j}^{2}+\left(1-u_{i v}\right) M^{2} \\
\forall i \in N \quad \sum_{j \in U} w_{i j}=1 \\
\forall v \in V \quad \sum_{i \in N} u_{i v} \geq 1 \\
\forall i \in N \quad \sum_{v \in V} u_{i v} \geq y_{i} \\
\forall i \in N \quad \sum_{v \in V} u_{i v} \leq|V| y_{i} .
\end{aligned}
$$

This is a nonconvex Mixed-Integer Nonlinear Programming (MINLP) problem with no objective function. Although some solution methods exist, this is amongst the most difficult problem classes ever to be tackled. It must be added that in this case the number of binary variables is considerable, making the problem all the more difficult to solve in practice.

\subsection{Approximation guarantee}

We remark that the reformulation of Sect. 3 is not exact. The fraction $\alpha$ of $T$ actually covered by the configuration of spheres solving $\left(P_{1}^{\prime}\right)$ depends on $\underline{r}=\min R$ and $\Delta$. The value of $\alpha$ can be estimated by computing the sphere density $\tau$ of the configuration, i.e. the fraction of the fundamental lattice region covered by spheres averaged on the whole lattice. It is easy to see that $\alpha \rightarrow 1$ for $\Delta \rightarrow 0$, as we recover the exact formulation $P_{1}$ "in the limit" as the lattice points grow closer. By the regularity of the functions involved in the formulation, the approximation guarantee on $\alpha$ can be made as small as desired by adding lattice points. On the other hand, this increases the number of variables in $P_{1}^{\prime}$ to such an extent that computational solutions become practically impossible to obtain: thus, $\underline{r}$ may have the same order of magnitude as $\Delta$, which means that estimating $\alpha$ in the worst case becomes important.

The worst case configuration minimizes $\alpha$ whilst covering all the points in the cubic lattice. This yields a configuration where all the spheres have radius $\underline{r}$ (to minimize the volume) and cover as many points as possible (to cover all the lattice points with as few spheres as possible). We remark that the fact that in $P_{1}^{\prime}$ the number of spheres is fixed is not troublesome: we assume that surplus spheres are superpositioned to other spheres in the configuration (this does not change the density or the covering properties of the current configuration). Naturally we assume that $P_{1}^{\prime}$ has at least one feasible solution so we do not need to deal with the case where there are not enough spheres to cover all the lattice points in $P \cap T$. Among all possible such configurations, we may assume some regularity in spherical placements without loss of generality, for irregularity may help cover more volume of a particular lattice cube at the expense of the adjacent ones, so the density throughout the lattice does not change. In the rest of this section, we shall study some worst case sphere densities for some (small) values of $\underline{r}$.

Since in each case we assume the worst case configuration to be the one with minimum possible sphere volume (i.e. using spheres of minimum radius), we only consider configurations where the spheres have radii $\underline{r}$ in a critical set $R^{*} \subseteq R$ defined as follows: for all $r^{*} \in R^{*}$ a sphere of radius $r \geq r^{*}$ can cover more lattice points than a sphere of radius $r<r^{*}$. The 
densities corresponding to values in the critical set are called critical densities. We define a function $\xi: R^{*} \rightarrow \mathbb{N}$ that maps critical radii to the maximum number of lattice points that can be covered by a sphere of the specified radius. We consider a subset $\bar{R} \subset R^{*}$ of small critical radii $\bar{R}=\frac{\Delta}{2}\{1, \sqrt{2}, \sqrt{3}, \sqrt{5}, 2 \sqrt{2}, 2 \sqrt{3}\}$, to which there correspond $\xi$ values of:

\begin{tabular}{c|cccccc}
$2 r^{*} / \Delta$ & 1 & $\sqrt{2}$ & $\sqrt{3}$ & $\sqrt{5}$ & $2 \sqrt{2}$ & $2 \sqrt{3}$ \\
\hline$\xi\left(r^{*}\right)$ & 2 & 4 & 8 & 10 & 19 & 27
\end{tabular}

Lemma 1 The sphere density $\tau$ corresponding to $2 r^{*} / \Delta=1$ is bounded above by $\frac{\pi}{6}$.

Proof In a sphere covering for a 3D cubic lattice having fundamental element width $\Delta$, for all $r<r^{*}=\frac{\Delta}{2}$ the diameter of spheres of radius $r$ is strictly less than $\Delta$, which means that no sphere can ever cover more than one point of $P$. Consider the covering consisting of pairwise-disjoint spheres centered at the lattice points. It is easy to show that this covering has a minimum number of spheres. In this covering, each lattice cube has 8 points each of which is covered by one spherical orthant (1/8-th of a sphere). Thus the configuration has one sphere per lattice cube. The sphere density for the limiting case $r=r^{*}$ is then $\tau=\frac{4 \pi\left(r^{*}\right)^{3}}{3(\Delta)^{3}}$, which is bounded above by $\frac{\pi}{6}$.

Lemma 2 The sphere density $\tau$ corresponding to $2 r^{*} / \Delta=\sqrt{2}$ is bounded above by $\frac{\pi}{12}(5-$ $2 \sqrt{2})$.

Proof A sphere covering of a 3D cubic lattic with unit element width $\Delta$ and sphere radii $r<$ $r^{*}=\frac{\sqrt{2}}{2} \Delta$ can cover at most 4 lattice points (see Fig. 1-left; the proof is simple). Assuming a regular and symmetric arrangement of the covering spheres, there are only two possible coverings where each sphere covers 4 lattice points, depicted in Fig. 1 (lattice of type L on the left and $\mathrm{R}$ on the right). A simple computation shows that $\alpha$ is minimum in the covering of type $\mathrm{L}$, which is therefore the worst case. The density of the covering of type $\mathrm{L}$ where the spheres have the limiting case of radius $r=r^{*}$ can be computed by considering the volume $z\left(\Delta, r^{*}\right)$ of the sphere crown determined by the radius ranging in $\left[\frac{\Delta}{2}, r^{*}\right]$, i.e. $\int_{(\Delta) / 2}^{r^{*}} \pi\left(\left(r^{*}\right)^{2}-\right.$ $\left.x^{2}\right) d x$. In the covering of type $\mathrm{L}$ there are two types of unit lattice elements: the "filled" ones (where two spheres cover a significant proportion of the element, see Fig. 1 left) and the "empty" ones. The covered volume in "filled" cubes is given by a whole sphere take away $6 z\left(\Delta, r^{*}\right)$ (4 per side and 2 where the two spheres intersect in the middle of the cube). For the "empty" cubes the covered volume is $2 z\left(\Delta, r^{*}\right)$. We obtain $\frac{1}{2 \Delta^{3}}\left(\frac{4}{3} \pi\left(r^{*}\right)^{3}-4 z\left(\Delta, r^{*}\right)\right)$, which is bounded above by $\frac{\pi}{12}(5-2 \sqrt{2})$.

In all other cases the technique is similar: find the worst case configuration, see how many lattice cubes it covers and in what way, and then divide the number of spherical volume elements by the number of lattice cubes that contains them. Approximate values for $\tau\left(r^{*}\right)$ are reported below.

\begin{tabular}{c|cccccc}
$2 r^{*} / \Delta$ & 1 & $\sqrt{2}$ & $\sqrt{3}$ & $\sqrt{5}$ & $2 \sqrt{2}$ & $2 \sqrt{3}$ \\
\hline$\tau\left(r^{*}\right)$ & 0.52 & 0.57 & 0.48 & 0.66 & 0.83 & 0.81
\end{tabular}

This table, and the fact that $\alpha \rightarrow 1$ as $\Delta \rightarrow 0$ or $\underline{r} \rightarrow \infty$, suggests that a ratio $\underline{r} / \Delta \geq 2$ should guarantee a fairly satisfactory covering of $T$. 

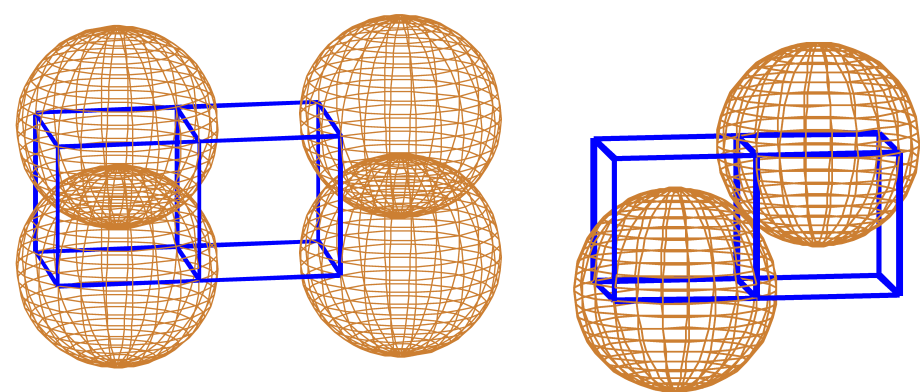

Fig. 1 Worst case sphere density configuration for $r^{*}=\frac{\sqrt{2}}{2} \Delta$, with density $\alpha \approx 0.5685$ (left). Alternative configuration for $r^{*}=\frac{\sqrt{2}}{2} \Delta$, with density $\alpha \approx 0.6115$ (right).

\section{Convex MINLP reformulation}

$\left(P_{1}^{\prime}\right)$ is a nonconvex MINLP. The state of the art in the solution methods for this class of problems $[2,12,15,19,6]$ is not at the stage where reasonably sized instances can be solved effectively or reliably. Although the nonconvexity in $\left(P_{1}^{\prime}\right)$ is caused by the set of binary products $u_{i v} w_{i j}$, which can all be linearized exactly in the standard way [7,13], this would imply adding $3|N||V||U|$ inequality constraints to the formulation. We employ a different reformulation: let $N=\{1, \ldots, n|R|\}$ and introduce a parameter $\rho: N \rightarrow \mathbb{R}_{+}$that assigns to each sphere a fixed radius in $R$. Since the set of spheres is now larger, we minimize the number of spheres actually used in the covering. The problem is transformed as follows.

$\left(P_{2}\right)$ Given a finite set of points $P \cap T \subset \mathbb{R}^{3}$, a finite set $R \subset \mathbb{R}_{+}$and index sets $N$ and $\rho: N \rightarrow R$, find a set of balls $\{B(x(i), \rho(i)) \mid i \in N\}$ of minimum cardinality covering each point in $P \cap T$.

The mathematical programming formulation for $\left(P_{2}\right)$ is the same as for $\left(P_{1}^{\prime}\right)$, with $(7)$ deleted, (6) replaced by

$$
\forall i \in N, v \in V \quad\left\|x_{i}-p_{v}\right\|^{2} \leq u_{i v} \rho_{i}^{2}+\left(1-u_{i v}\right) M^{2},
$$

and

$$
\min \sum_{i \in N} y_{i}
$$

as objective function. Namely, minimize (12) subject to (8)-(11). Since (11) is a convex constraint, the problem is now a cMINLP. Furthermore, we have eliminated the binary radius assignment variables $w$ (at the expense of enlarging $N$, of course - but this usually yields a computational improvement).

Reformulations that are linked to a change in the problem definition (and not only to the problem formulation) are called reformulations in the sense of Hansen $[1,14]$. We prove that the reformulation given above is a reformulation in the sense of Hansen. For an instance $I$ of a sphere covering problem $P,|I|$ is the size of the instance (i.e the number of bits necessary to encode all the values of the problem parameters), $n(I)$ is the number of spheres in the solution of $I$ and $N(I)$ is the set of sphere indices in instance $I$.

Proposition 1 Given a feasible instance $I_{1}$ of $\left(P_{1}\right)$, there is a feasible instance $I_{2}$ of $\left(P_{2}\right)$ (where $\left|I_{2}\right|$ is polynomial in $\left.\left|I_{1}\right|\right)$ such that if $(x, y, u)$ optimally solves $I_{2}$ then an optimal solution $\left(x^{\prime}, y^{\prime}, u^{\prime}, w^{\prime}\right)$ of $I_{1}$ can be obtained in linear time in $n\left(I_{2}\right)$. 
Proof Let $n\left(I_{2}\right)=|U| n\left(I_{1}\right)$, for all $j \in U, i \leq n\left(I_{1}\right)$ let $\omega(i, j)=(j-1) n\left(I_{1}\right)+i$, and define $\rho_{\omega(i, j)}=r_{j}$. If $I_{1}$ is feasible, $I_{2}$ is also feasible because for each potential sphere in $I_{1}$ there are $|U|$ potential spheres having all the possible radii in $R$. The size of $\left|I_{2}\right|$ is polynomial by construction. Let $(x, y, u)$ be an optimal solution of $I_{2}$. Since each sphere has a unique radius, there is an ordering of $N\left(I_{2}\right)$ such that $\sum_{j \in U} y_{\omega(i, j)} \leq 1$ for all $i \in N\left(I_{1}\right)$. Assume $N\left(I_{2}\right)$ is ordered in such a way. For all $i \in N\left(I_{1}\right), j \in U$ let $w_{i j}^{\prime}=y_{\omega(i, j)}$ if $\rho_{\omega(i, j)}=r_{j}$ and 0 otherwise; for $i \in N\left(I_{1}\right)$ let $y_{i}^{\prime}=\sum_{j \in U} y_{\omega(i, j)}$ and $x_{i}^{\prime}=\sum_{j \in U} y_{\omega(i, j)} x_{\omega(i, j)}$; and for $i \in N\left(I_{1}\right)$ and $v \in V$ let $u_{i v}^{\prime}=$ $\sum_{j \in U} y_{\omega(i, j)} u_{\omega(i, v), v}$. It follows immediately that $\sum_{i \in N\left(I_{1}\right)} y_{i}=\sum_{i \in N\left(I_{2}\right)} y_{i}^{\prime}$, which implies optimality of $\left(x^{\prime}, y^{\prime}, u^{\prime}, w^{\prime}\right)$ in $I_{1}$. It can be immediately verified that the optimal solution of $I_{1}$ can be obtained in time linear in $n\left(I_{2}\right)$.

\subsection{Inner approximation of the norm}

One relatively straightforward approximating reformulation that is sometimes applied to MINLPs is outer or inner approximation of wholly convex/concave terms. Problem $\left(P_{2}\right)$ is nonlinear because of the norm term $\left\|x_{i}-p_{v}\right\|^{2}$ in (11) for all $i \in N, v \in V$. Now, $\left\|x_{i}-p_{v}\right\|^{2}=$ $\sum_{k \leq 3}\left(x_{i k}^{2}-2 x_{i k} p_{v k}+p_{v k}^{2}\right)$, so the only nonlinear term is $x_{i k}^{2}$. We can replace it by an added linearization variable

(e) $s: N \rightarrow \mathbb{R}_{+}^{3}: s_{i k}$ replaces $x_{i k}^{2}$ for $i \in N, k \leq 3$,

and change (11) accordingly:

$$
\forall i \in N, v \in V \quad \sum_{k \leq 3}\left(s_{i k}-2 x_{i k} p_{v k}+p_{v k}^{2}\right) \leq u_{i v} \rho_{i}^{2}+\left(1-u_{i v}\right) M^{2}
$$

In order to make sure that the $s_{i k}$ variables take on values that are feasible in the quadratic constraint (11), we need $s_{i k} \geq x_{i k}^{2}$ for $i \in N, k \leq 3$. We employ the inner approximation depicted in Fig. 2 using a finite number of evenly spaced points $\bar{x}_{i k d}$ for $d$ in some suitable index set $D(|D|$ is the number of inner approximation points). Formally, we define:

$$
\forall i \in N, k \leq 3 \quad s_{i k}=\max \left\{\left(\bar{x}_{i k d}+\bar{x}_{i k, d-1}\right) x_{i k}-\bar{x}_{i k d} \bar{x}_{i k, d-1} \mid d \in D \backslash\{1\}\right\}
$$

Eq.(14) can be reformulated as a set of $|D|-1$ linear constraints

$$
\forall i \in N, k \leq 3, d \in D \backslash\{1\} \quad s_{i k} \geq\left(\bar{x}_{i k d}+\bar{x}_{i k, d-1}\right) x_{i k}-\bar{x}_{i k d} \bar{x}_{i k, d-1} .
$$

It is easy to show that $s_{i k} \geq x_{i k}^{2}$ for all $i \in N, k \leq 3$.

Formulation $\left(P_{2}^{\prime}\right)$ is like $\left(P_{2}\right)$ with the added variables $s$, added constraints $(15)$, and (11) replaced by (13). Namely, minimize (12) subject to (8)-(10), (13), (15). It is a MILP that approximates $\left(P_{2}\right)$. It is easy to see that for $x_{i k d}-x_{i k, d-1} \rightarrow 0$ the MILP becomes a SemiInfinite Programming (SIP) problem which is an exact SIP reformulation of the cMINLP $\left(P_{2}\right)$. We remark that this type of approximation can also be applied to $\left(P_{1}^{\prime}\right)$, yielding a MILP $\left(P_{1}^{\prime \prime}\right)$ obtained in the same way as $\left(P_{2}^{\prime}\right)$.

Formulation $\left(P_{2}^{\prime}\right)$ can be solved by CPLEX [9]. The practical performance ranges from reasonable to unsatisfactory. 


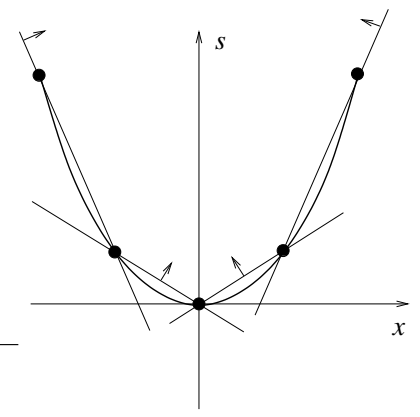

Fig. 2 Inner approximation of $x^{2}$.

\section{MILP approximation}

One way to get rid of the problematic "big M" constraint (6) is to encode all possible distances $\left\|x_{i}-p_{v}\right\|$ (for $i \in N, v \in V$ ) into the problem instance, and reformulate this to a pure covering problem. Since there is an uncountable number of spatial positions for $x_{i}$, we approximate the problem by requiring that the sphere centers may only be positioned on the lattice points. For all $i \in N, v \in V$ we introduce the sets $\eta_{i v} \subset V$ describing the covering extent of the $i$-th sphere for the lattice poing $v$, i.e. $\eta_{i v}=\left\{t \in V \mid\left\|p_{v}-p_{t}\right\| \leq \rho_{i}\right\}$. As the sphere centers are now fixed, we replace the variables $x$ by variables $\chi$ :

(a') $\chi: N \times V \rightarrow\{0,1\}$ is set to 1 if the given sphere is centered on the specified lattice point, subject to assignment constraints

$$
\forall i \leq N \quad \sum_{v \in V} \chi_{i v}=y_{i}
$$

The covering conditions become:

$$
\begin{gathered}
\forall i \in N, v \in V, t \in \eta_{i v} \quad u_{i v} \geq \chi_{i v} \\
\forall i \in N, v \in V \quad \sum_{\substack{t \in V \\
v \in \eta_{i t}}} \chi_{i t} \geq u_{i v} .
\end{gathered}
$$

This is a MILP formulation describing the following problem.

$\left(P_{3}\right)$ Given a finite set of points $P \cap T \subset \mathbb{R}^{3}$ indexed by the index set $V$, an index set $N$, and a set-valued function $\eta: N \times V \rightarrow \mathscr{P}(V)$ (the power set of $V$ ), find a function $\chi: N \times V \rightarrow\{0,1\}$ such that the set $S=\left\{(i, v) \mid \chi_{i v}=1\right\}$ has minimum cardinality and has the property that for all $t \in V$ there is $(i, v) \in S$ with $t \in \eta_{i v}$.

We remark that the approximation analysis of Sect. 3.1 is not applicable in this case. The fact that $\alpha \rightarrow 1$ as $\Delta \rightarrow 0$, however, still holds.

\section{Minimizing radiation on healthy tissues}

Although it is of course of the utmost importance to cover as much as possible of the tumor with radiation, it is also desirable not to "overshoot" excessively: the spheres placed near the 
border of $T$ will in general cover a region of healthy brain tissue, which must be minimized. To this aim we consider a band-shaped region (of width $\bar{r}$ ) around $T$ and define the set $\bar{T}=\left\{x \in \mathbb{R}^{3} \mid \exists x^{\prime} \in T\left(\left\|x-x^{\prime}\right\|<\bar{r}\right)\right\}$ that contains the tumor and a region of healthy brain tissue around it. We define a larger cubic lattice set $\bar{P}$ (the smallest such lattice containing $\bar{T}$ ), define $\bar{V}$ to be a set of integers indexing the points in $\bar{P} \cap \bar{T}$ and extend $u_{i v}$ to be defined for each $i \in N$ and for each $v \in \bar{V}$.

These changes reflect on $\left(P_{2}^{\prime}\right)$ and $\left(P_{3}\right)$ differently. For $\left(P_{2}^{\prime}\right)$, we reformulate to $\left(P_{2}^{\prime \prime}\right)$ as follows: we quantify (13) and change the sum quantifiers of (9)-(10) to $\bar{V}$ instead of $V$ and modify the objective function (12) to:

$$
\min \sum_{i \in N}\left(y_{i}+\frac{1}{|\bar{V}|-|V|} \sum_{v \in \bar{V} \backslash V} u_{i v}\right) .
$$

Namely, minimize (19) subject to (8)-(10), (13), (15) with $V$ replaced by $\bar{V}$. For $\left(P_{3}\right)$, we reformulate to $\left(P_{3}^{\prime}\right)$ as follows: we extend $\eta$ to be defined on $\bar{V}$, change the sum quantifiers of (16) to $\bar{V}$ instead of $V$, change (18) to

$$
\forall i \in N, v \in \bar{V} \quad \sum_{\substack{t \in V \\ v \in \eta_{i t}}} \chi_{i t} \geq u_{i v}
$$

and employ the objective function (19) above. Namely, minimize (19) subject to (16) with $V$ replaced by $\bar{V},(17),(20)$.

\section{Computational results}

In this section we present computational results for formulations $P_{1}^{\prime}, P_{1}^{\prime \prime}, P_{2}, P_{2}^{\prime \prime}$, and $P_{3}^{\prime}$. Computational results for $P_{2}^{\prime}$ (resp. $P_{3}$ ) are comparable with those of $P_{2}^{\prime \prime}\left(\right.$ resp. $P_{3}^{\prime}$ ). As the latter include considerations from Sect. 6, they are more realistic.

All computational results were obtained on a PC with an Intel Core Duo 1.2GHz CPU with 1.5GB RAM running Linux, under the AMPL [8] modelling environment. Nonconvex MINLPs $\left(P_{1}^{\prime}\right)$ were solved by using a VNS algorithm [15] coded in AMPL and tailored for MINLPs (with maximum neighbourhood limit set to 10), with minlp_bb [11] (v. 20020703) as local solver; this was used by itself to solve cMINLPs $\left(P_{2}\right)$. MILPs $\left(P_{1}^{\prime \prime}, P_{2}^{\prime \prime}, P_{3}^{\prime}\right)$ were solved by CPLEX 10.1 [9]. Solution quality is established by three parameters: CPU time (expressed in seconds of user time) taken to obtain a solution, an estimation $\alpha$ of the fraction of tumor volume covered by the spheres ( $\alpha$ is computed by using a very fine lattice grid covering $T$ ), and the number $\mathbf{y}$ of spheres used in the covering.

All tested instances are based on realistic machine settings with respect to the set $R ; N$ was set to 5 for problems $\left(P_{1}^{\prime}, P_{1}^{\prime \prime}\right)$ and to $5|R|=20$ for problems $\left(P_{2}, P_{2}^{\prime \prime}, P_{3}^{\prime}\right)$. Each instance is then described by a 5-tuple $\left(r_{T}^{x}, r_{T}^{y}, r_{T}^{z}, \bar{r}, \Delta\right)$ specifying the radii of the ellipsoid-shaped tumor, the healthy band width, and the side length of the cubic fundamental lattice region. All instances for $P_{1}^{\prime \prime}, P_{2}^{\prime \prime}$ have $|D|=10$ in the linearization of the square term (Sect. 4.1). Instance statistics such as the total number of variables (var), split into binary (bin) and continuous (var - bin), constraints (con) and nonlinear terms (nlt) are reported in Table 1. All statistics have been recorded after any AMPL presolver simplifications.

The computational results in Table 2 show that all models suffer from different types of blemishes. Most notably, all models except $\left(P_{3}^{\prime}\right)$ take too much CPU time to find a solution. $\left(P_{3}^{\prime}\right)$, however, does not find solutions with high $\alpha$ values (as is to be expected as $\alpha$ is linked to the sphere density $\tau$, and the approximation results for $\tau$ do not hold in $P_{3}^{\prime}$ ). We remark 


\begin{tabular}{|c||c|c|c|c|c||c|c|c|c|c|c|c|}
\hline & & & & & & \multicolumn{3}{|c|}{$P_{1}^{\prime}$} & \multicolumn{3}{|c|}{$P_{1}^{\prime \prime}$} \\
\hline Name & $r_{T}^{x}$ & $r_{T}^{y}$ & $r_{T}^{z}$ & $\bar{r}$ & $\Delta$ & var & bin & con & nlt & var & bin & con \\
\hline bt1 & 6 & 8 & 12 & 4 & 5 & 919 & 904 & 918 & 9625 & 559 & 529 & 1478 \\
bt2 & 6 & 8 & 12 & 4 & 3 & 3509 & 3494 & 3576 & 38115 & 2259 & 2229 & 5966 \\
bt3 & 6 & 8 & 12 & 4 & 2 & 12199 & 12184 & 12465 & 133705 & 7234 & 7204 & 19100 \\
bt4 & 10 & 10 & 10 & 6 & 3 & 6699 & 6684 & 6832 & 73205 & 3909 & 3879 & 10322 \\
\hline \multicolumn{10}{|c|}{$P_{2}$} \\
\hline Name & var & bin & con & nlt & var & bin & con & var & bin & con \\
\hline bt1 & 3580 & 3520 & 3560 & 10500 & 3640 & 3520 & 4100 & 1550 & 1550 & 4790 \\
bt2 & 13940 & 13880 & 13968 & 41580 & 14000 & 13880 & 14528 & 8480 & 8480 & 87543 \\
bt3 & 48700 & 48640 & 48947 & 145860 & 48760 & 48640 & 49487 & 31090 & 31090 & 886877 \\
bt4 & 26700 & 26640 & 26814 & 79860 & 26760 & 26640 & 27354 & 13160 & 13160 & 154819 \\
\hline
\end{tabular}

Table 1 Instance statistics.

that the failure noted for CPLEX on the bt 4 instance for $P_{1}^{\prime \prime}$ is to be interpreted as not finding a feasible solution after over $7 \mathrm{~h}$ of $\mathrm{CPU}$ time.

\begin{tabular}{|c|c|c|c|c|c|c|c|c|c|c|c|c|}
\hline & & \multicolumn{4}{|c|}{$P_{1}^{\prime}(\mathrm{VNS})$} & \multicolumn{5}{|c|}{$P_{1}^{\prime \prime}$ (CPLEX) } & & \\
\hline & Name & $\mathrm{CPU}$ & & $\alpha$ & $\mathbf{y}$ & & $\mathrm{CPU}$ & & $\alpha$ & $\overline{\mathbf{y}}$ & & \\
\hline & bt1 & 2682 & & .963 & 2 & & $1407^{*}$ & & 0.874 & 2 & & \\
\hline & bt2 & 15807 & & $967^{\dagger}$ & 4 & 511 & $68(50 \%)$ & & 0.984 & 5 & & \\
\hline & bt3 & (fail) & & - & - & 542 & $17(75 \%)$ & & 0.999 & 4 & & \\
\hline & bt4 & 22701 & & $426^{\dagger}$ & 2 & & (fail) & & - & - & & \\
\hline & \multicolumn{3}{|c|}{$P_{2}$ (minlp_bb) } & \multicolumn{5}{|c|}{$\begin{array}{l}P_{2}^{\prime \prime} \text { (CPLEX) } \\
\end{array}$} & \multicolumn{4}{|c|}{$\begin{array}{l}P_{3}^{\prime} \text { (CPLEX) } \\
\end{array}$} \\
\hline Name & $\mathrm{CPU}$ & $\alpha$ & $\mathbf{y}$ & & $\mathrm{CPU}$ & & $\alpha$ & $\mathbf{y}$ & $\mathrm{CPL}$ & & $\alpha$ & $\mathbf{y}$ \\
\hline bt1 & 847.6 & $0.158^{\dagger}$ & 4 & & $357^{*}$ & & 0.84 & 2 & 0.26 & & 0.75 & 5 \\
\hline bt 2 & (fail) & - & - & 728 & $7(239$ & & 0.981 & 2 & 13.23 & & 0.71 & 2 \\
\hline bt3 & (fail) & - & - & 600 & $8(66 \%$ & & 1 & 2 & 8701 & & 0.91 & 2 \\
\hline bt4 & (fail) & - & - & 586 & $8(76 \%$ & & 0.907 & 3 & 3206 & & 0.78 & 3 \\
\hline
\end{tabular}

Table 2 Computational results. Times marked with * correspond to CPLEX running to optimality. Values marked with ${ }^{\dagger}$ correspond to infeasible runs.

These computational results also validate the reformulation-based methodological approach: the resulting formulations (i.e. $P_{2}^{\prime \prime}$ and $P_{3}^{\prime}$ ) are easier to solve and/or provide better quality solutions than their predecessors in the reformulation sequence.

\section{Conclusion}

This paper discusses a sphere covering problem arising in the configuration of a gamma ray machine for the cure of brain tumor. We employ a solution methodology based on successive reformulations going from a natural infinite formulation through nonconvex mixed-integer nonlinear and convex mixed-integer nonlinear to two very different types of mixed-integer linear formulations. We also report on preliminary computational results. Work is under way in investigating different types of formulations based on bounding interspherical distance from below. The final aim is that of integrating the modelling relative to the dosage problem and thus of proposing formulations for the whole treatment problem. 


\section{References}

1. Audet, C., Hansen, P., Jaumard, B., Savard, G.: Links between linear bilevel and mixed 0-1 programming problems. Journal of Optimization Theory and Applications 93(2), 273-300 (1997)

2. Brook, A., Kendrick, D., Meeraus, A.: Gams, a user's guide. ACM SIGNUM Newsletter 23(3-4), 10-11 (1988)

3. Ferris, M., Lim, J., Shepard, D.: An optimization approach for the radiosurgery treatment planning. SIAM Journal on Optimization 13(3), 921-937 (2003)

4. Ferris, M., Shepard, D.: Optimization of gamma knife radiosurgery. In: D.Z. Du, P. Pardalos, J. Wang (eds.) Discrete Mathematical Problems with Medical Applications, DIMACS Series in Discrete Mathematics and Theoretical Computer Science, vol. 55, pp. 27-44. AMS (2000)

5. Ferris, M., Voelker, M.: Neuro-dynamic programming for radiation treatment planning. Annals of Operations Research 119, 247-260 (2003)

6. Floudas, C.: Deterministic Global Optimization. Kluwer Academic Publishers, Dordrecht (2000)

7. Fortet, R.: Applications de l'algèbre de boole en recherche opérationelle. Revue Française de Recherche Opérationelle 4, 17-26 (1960)

8. Fourer, R., Gay, D.: The AMPL Book. Duxbury Press, Pacific Grove (2002)

9. ILOG: ILOG CPLEX 10.1 User's Manual. ILOG S.A., Gentilly, France (2006)

10. Jitprapaikulsarn, S.: An optimization-based treatment planner for gamma knife radiosurgery. Ph.D. thesis, Case Western Reserve University (2005)

11. Leyffer, S.: User manual for minlp_bb. Tech. rep., University of Dundee, UK (1999)

12. Liberti, L.: Writing global optimization software. In: Liberti and Maculan [16], pp. 211-262

13. Liberti, L.: Compact linearization of binary quadratic problems. 4OR 5(3), 231-245 (2007)

14. Liberti, L.: Reformulation techniques in mathematical programming (2007). Thèse d'Habilitation à Diriger des Recherches

15. Liberti, L., Dražic, M.: Variable neighbourhood search for the global optimization of constrained nlps. In: Proceedings of GO Workshop, Almeria, Spain (2005)

16. Liberti, L., Maculan, N. (eds.): Global Optimization: from Theory to Implementation. Springer, Berlin (2006)

17. Lim, J.: Optimization in radiation treatment planning. Ph.D. thesis, University of Wisconsin - Madison (2002)

18. Olafsson, A., Wright, S.: Linear programming formulations and algorithms for radiotherapy treatment planning. Optimization Methods and Software 21(2), 201-231 (2004)

19. Tawarmalani, M., Sahinidis, N.: Convexification and Global Optimization in Continuous and MixedInteger Nonlinear Programming : Theory, Algorithms, Software, and Applications. Kluwer, Dordrecht (2002) 